\title{
Cyclic Guanosine Monophosphate as a Mediator of Vasodilation
}

\author{
Ferid Murad \\ Departments of Medicine and Pharmacology, Stanford University and Veterans Administration Medical Center, \\ Palo Alto, California 94304
}

Although cyclic guanosine monophosphate (GMP) ${ }^{1}$ was first described in biological samples more than two decades ago, its role in some physiological processes has only become apparent in the past few years (see references 1-4). This relatively slow development is probably attributable to the low concentrations of the nucleotide in tissues, the complex and insensitive methods available during the early studies, and the biases many investigators had regarding its possible functions. The latter was undoubtedly influenced by the many similarities of the cyclic GMP system with that of cyclic AMP and the attention cyclic AMP has received during this period. While analogies and similarities between these two cyclic nucleotide systems do exist, the cyclic GMP system presents more complexities due to the existence of several isoenzymes responsible for its synthesis.

It is known that the conversion of guanosine triphosphate (GTP) to cyclic GMP is catalyzed by at least two isoenzyme forms of guanylate cyclase. The kinetic, physicochemical, and antigenic properties of the cytosolic and membrane-associated isoenzymes are quite different (see references 2,4$)$. The relative abundance of the soluble and particulate enzyme is variable in different tissues and species. While intestinal mucosa and retina possess predominately the particulate isoenzyme and platelets contain the soluble isoenzyme, most tissues such as vascular smooth muscle have both isoenzymes. Furthermore, the regulation of each of these isoenzymes is quite different. The soluble enzyme appears unique in that it can be activated by reactive free radicals such as nitric oxide (5), and probably hydroxyl free radical $(6)$ and some porphyrins $(7,8)$. On the other hand, the particulate isoenzyme can be activated with agents such as Escherichia coli heat-stable enterotoxin $(9-11)$, atriopeptins $(12,13)$, and hemin (14). Cations, thiols, other redox agents, and detergents also have complex effects on the activity of both isoenzymes (2).

Studies with the kinetic characterization of these isoenzymes led to the present understanding of the role of cyclic GMP in smooth muscle relaxation. Azide, added to inhibit GTPase activity in crude enzyme preparations, was found to activate the enzyme (15). While some hormones, autocoids, and other agents were able to increase cyclic GMP accumulation in intact tissues, these agents had no effects on guanylate cyclase activity in broken

Received for publication 9 January 1986.

1. Abbreviations used in this paper: EDRF, endothelial-derived relaxant factor; GMP, guanosine monophosphate; GTP, guanosine triphosphate; ST, Escherichia coli heat-stable enterotoxin.

J. Clin. Invest.

(c) The American Society for Clinical Investigation, Inc.

0021-9738/86/07/0001/05 \$1.00

Volume 78, July 1986, 1-5 cell preparations. It was reasoned that an understanding of azide activation of guanylate cyclase could lead to understanding the mechanisms of hormonal regulation of the enzyme and some functions of cyclic GMP. Some of these predictions have been fulfilled. The stimulatory effects of azide were dependent upon the presence of heme-containing proteins in guanylate cyclase incubations such as catalase, peroxidase, or cytochromes (16). In addition to azide, other agents that are capable of generating nitric oxide in incubations also activated the enzyme and included hydroxylamine, sodium nitrite, nitroglycerin, sodium nitroprusside $(15,17)$, and nitrosamines (18). Most of these agents also increased cyclic GMP accumulation in various intact tissue preparations including smooth muscle segments (19-22). The increase in cyclic GMP with these guanylate cyclase activators was associated with relaxation of tracheal, intestinal, and vascular smooth muscle $(20,22,23)$. These studies led to the first proposed role of cyclic GMP, regulation of smooth muscle relaxation, that has withstood the test of time (22). These and subsequent studies with cyclic GMP in $E$. coli heat-stable enterotoxin-induced diarrhea (9-11) and light-induced ion conductance in the retina (24) have further stimulated the interest in cyclic GMP and the search for other functions.

We have coined the term "nitrovasodilators" for those agents that can lead to the formation of the reactive nitric oxide-free radical in incubations and increase cyclic GMP synthesis. These agents include the organic nitrates such as nitroglycerin, the inorganic nitrates and nitrites such as nitroprusside and sodium nitrite, various nitrosoureas and nitrosamines, as well as azide, hydroxylamine, and perhaps some hydrazines. Some of these nitrovasodilators require catalytic conversion to nitric oxide by a macromolecule or enzyme while others are nonenzymatically converted to nitric oxide under the appropriate oxidizing or reducing conditions. The effects of $\mathrm{pH}$ and oxygen tension on the formation of nitric oxide from a precursor and the metabolism of these nitrovasodilators may explain their apparent specificity for different vascular beds and tissues. However, this hypothesis requires additional investigation. Activation of guanylate cyclase and cyclic GMP accumulation with these nitric oxide-generating precursors occurs at relatively low concentrations (micromolar to nanomolar range) and is a function of the presence of other compounds that act as sinks, or reduce or oxidize the formed nitric oxide. Activation of the enzyme is instantaneous without a temporal lag and is reversible $(5,17)$. While the precise mechanism of activation of the enzyme is not presently understood, critical thiol groups on the enzyme $(25,26)$ and heme $(7,27)$ which may be a prosthetic group on the enzyme (28) appear to participate in the activation mechanism. Clearly, homogeneous preparations of the soluble isoenzyme can be activated by these nitrovasodilators under the appropriate conditions, and the concentration of nitric oxide required for half-maximal activation of purified enzyme is estimated to be in the nanomolar 
range $(5,25)$. While crude particulate preparations of guanylate cyclase are also activated, this may be attributable to entrapped soluble isoenzyme that is virtually always present in the absence of some purification steps to remove it. Unfortunately, detergents prevent activation of either isoenzyme by a variety of agents. Thus, the issue of contaminating soluble isoenzyme contributing to the apparent activation of the particulate isoenzyme can not be presently resolved with purification procedures to separate the isoenzymes.

Agents that interfere with the conversion of the nitrovasodilator precursor to nitric oxide, react with nitric oxide, or inhibit guanylate cyclase activation decrease cyclic GMP accumulation in smooth muscle and shift dose-response (relaxation) curves to the right. This list includes various nonspecific as well as relatively specific antagonists such as cystamine, cystine, methylene blue, hemoglobin, methemoglobin, and cyanide $(15,17,27)$. While these agents have been useful in many in vitro systems to implicate cyclic GMP in some process, their nonspecific effects limit their usefulness at present in most in vivo systems. Agents that inhibit cyclic GMP hydrolysis by cyclic nucleotide phosphodiesterases predictably shift the dose-response curves of these nitrovasodilators to the left $(20,29)$. These antagonistic and synergistic effects should be important in predicting various drug interactions and modifying the therapeutic responses to the nitrovasodilators. For example, studies in dogs have shown that some of the cardiovascular effects of nitroprusside can be enhanced with aminophylline (30). The effects of ouabain to inhibit nitrovasodilator-induced cyclic GMP synthesis and vascular relaxation (31) should alert us to potentially important drug interactions between nitrovasodilators and cardiac glycosides which are commonly used together. The precise mechanisms by which inhibition of the Na-K pump with cardiac glycosides decreases cyclic GMP synthesis as well as cyclic GMP-induced vascular relaxation are unknown (31). Calcium availability for these processes, however, should be suspected as a candidate in these effects. Although the activation of guanylate cyclase and the accumulation of cyclic GMP in tissues with nitrovasodilators is calcium-independent (20), calcium antagonists may modify some of the physiological processes regulated by cyclic GMP and its rate of hydrolysis by phosphodiesterase. Indeed, the smooth muscle relaxant effects of the nitrovasodilators and calcium antagonists would be expected to be additive.

The increase in cyclic GMP in tracheal and vascular smooth muscle is associated with the activation of cyclic GMP-dependent protein kinase and the phosphorylation of numerous smooth muscle proteins (32-35). One of the smooth muscle proteins in which phosphorylation is decreased with nitrovasodilators or cyclic GMP analogues is myosin light chain $(3,35,36)$. While the phosphorylation of other smooth muscle proteins is also altered, their identities and roles are presently unknown. The mechanism of cyclic GMP-induced dephosphorylation of myosin light chain is also presently unknown. These effects could result from the decreased activity of myosin light chain kinase, a calcium and calmodulin-dependent enzyme, increased activity of the phosphatase, or a combination of these effects. The recent demonstration of an effect of nitroglycerin on Quin-2 fluorescence in smooth muscle cells (37) suggests that these effects could be due to decreased cytosolic calcium concentrations.

Although azide, nitroprusside, and other nitrovasodilators have been useful agents in some systems to evaluate the role of cyclic GMP in some process, their inhibition of metabolic pro- cesses with high concentrations and their oxidation of some ligands and hormones can also lead to artifacts and erroneous conclusions regarding functions of cyclic GMP.

The recent studies of Furchgott and his associates (38) have permitted us to understand the mechanism of action of another class of vascular relaxants, the endothelium-dependent vasodilators. Relaxation of blood vessels from a variety of species by agents such as acetylcholine, histamine, bradykinin, ionophore A23187, thrombin, and ATP requires the integrity of the vascular endothelium (see references 3, 38). These endothelium-dependent vasodilators either have no effect or may cause vasoconstriction in the absence of the endothelium. The latter effects are dependent upon the vessel and species being examined. These agents interact with specific receptors on endothelial cells to induce the formation and release of a factor(s) called endothelialderived relaxant factor (EDRF). The structure of this reactive and labile material is not known due to its short half-life and probably low concentration $(38,39)$. While studies with inhibitors of phospholipase and lipoxygenase have suggested that the factor may be an oxidized ecosinoid product (see references 3 , 38 ), the lack of specificity of these inhibitors limits such conclusions. Furthermore, it is not presently known if the various endothelium-dependent vasodilators induce the release of the same or different factors.

Relaxation of vascular segments with this class of vasodilators is also associated with guanylate cyclase activation and cyclic GMP accumulation in vascular smooth muscle $(35,36,40-42)$. Since nitroglycerin-tolerant vessels have decreased cyclic GMP accumulation and relaxation to the endothelium-dependent vasodilators as well as the nitrovasodilators (43), it appears that activation of soluble guanylate cyclase also mediates the effects of EDRF. Effects of EDRF on the particular isoenzyme, however, can not be excluded. The increased synthesis of cyclic GMP with endothelium-dependent vasodilators is also associated with cyclic GMP-dependent protein kinase activation and dephosphorylation of myosin light chain $(32,36)$. Indeed, the profiles of altered protein phosphorylation with two-dimensional polyacrylamide gel electrophoresis of extracts from vascular smooth muscle after treatment with nitrovasodilators, endothelium-dependent vasodilators, and 8-bromo cyclic GMP are similar (3436). While these effects with nitrovasodilators and 8-bromo cyclic GMP are endothelium-independent, the effects of endotheliumdependent vasodilators require the endothelium. Thus, the cascade of events distal to guanylate cyclase activation and cyclic GMP synthesis appear to be identical with both classes of vasodilators (see Fig. 1). These studies suggest that EDRF could be viewed as the endogenous equivalent of the nitrovasodilators or the "endogenous nitrate". The effects of nitric oxide and EDRF on guanylate cyclase activity and the inhibition of their effects with similar classes of agents such as hemoglobin, methylene blue, cyanide, and various reducing agents suggest that these reactive materials may also activate the enzyme by similar mechanisms (2-4, 44, 45). However, additional kinetic and physicochemical studies with purified enzyme and activators are required.

In the presence of the endothelium the basal levels of cyclic GMP and the activity of cyclic GMP-dependent protein kinase are increased, which suggests that basal release of EDRF occurs and can influence vascular motility $(32,36,40,41)$. The decreased basal or stimulated release of EDRF with endothelial damage could obviously have important clinical and therapeutic 


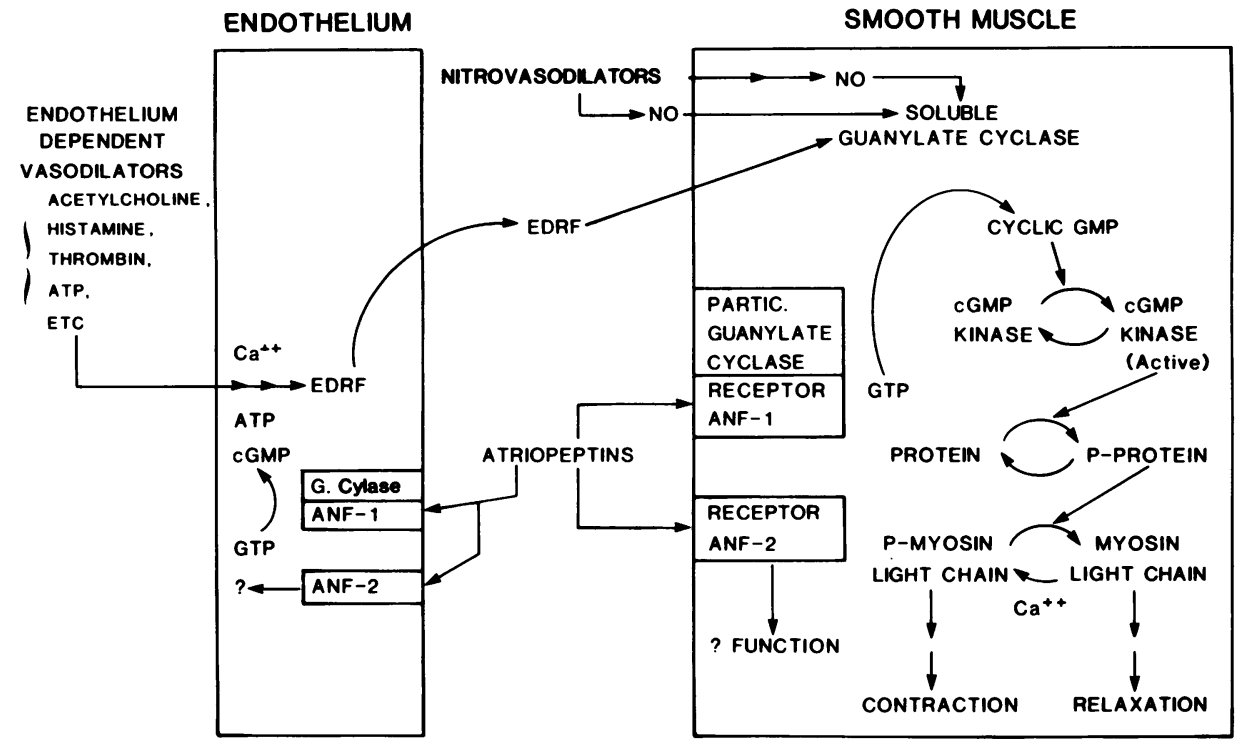

Figure 1. Proposed mechanism of action of some vasodilators on cyclic GMP synthesis and vascular relaxation. Through specific receptors on endothelial cells, a variety of endothelium-dependent vasodilators lead to the synthesis and release of EDRF. The structure of EDRF is unknown. Nitric oxide (NO) derived from the nitrovasodilators, and EDRF activate the soluble isoenzyme form of guanylate cyclase and result in increased cyclic GMP synthesis and cyclic GMP-dependent protein kinase activation. These events result in the dephosphorylation of myosin light chain and relaxation. Atriopeptins, through a specific receptor designated ANF-1, result in the activation of the membrane-associated isoenzyme form of guanylate cyclase, cyclic GMP synthesis, and cyclic GMP-dependent protein kinase activation. The function of the receptor that is not coupled to cyclic GMP synthesis (ANF-2) is unknown. While the atriopeptins relax vascular smooth muscle in the absence of the endothelium, the endothelial cells also possess atriopeptin receptors that are coupled to cyclic GMP synthesis. The function of cyclic GMP in endothelial cells is presently unknown.

implications in many cardiovascular disorders. Indeed, increased contraction or spasm at sites of intimal damage would be expected to occur due to the loss of EDRF and decreased cyclic GMP synthesis. Furthermore, with intimal damage, endothelium-dependent vasodilators should also be less effective than therapeutic agents that act in an endothelium-independent manner such as nitrovasodilators and atriopeptins (see below). Since the synthesis and release of EDRF as well as smooth muscle contraction is calcium-dependent, the interactions of calcium antagonists with these agents on cyclic GMP levels and relaxation are more complicated. We hope that the ability to assay EDRF in media from endothelial cell cultures (39) will facilitate the characterization and identification of this important substance and could lead to a new class of therapeutic vasodilators.

A third class of vasodilators that appear to mediate their effects through cyclic GMP synthesis is the atriopeptins $(12,13$, 45). This newly described group of peptide hormones has a number of cardiovascular and renal effects including natriuresis, diuresis, vasodilation, and inhibition of aldosterone secretion (46-48). This family of peptide hormones is derived from a precursor peptide or preprohormone of 152 amino acids found in granules in the cardiac atria $(49,50)$. The presence of the hormone and/or messenger RNA for its synthesis in other tissues as well as atria suggest that these peptides may have many other functions not presently appreciated. For example, it is suspected that these peptides may also serve as neurotransmitters as well as peripheral hormones.

The effects of atriopeptins on ion and water transport in the kidney are reminiscent of the effects of $E$. coli heat-stable enterotoxin (ST) in intestinal mucosa. Since the effects of ST are mediated through guanylate cyclase activation and cyclic GMP accumulation (9-11), we were prompted to examine the effects of atriopeptins on cyclic GMP metabolism in renal tissue, vascular tissue, and other tissues $(12,13,51,52)$. This class of peptide hormones specifically activates the particulate isoenzyme form of guanylate cyclase in many tissues including blood vessels, kidney, adrenal, and cultures of endothelial and smooth muscle cells $(12,13,51,52)$. The effects of atriopeptins on particulate guanylate cyclase are mediated through a specific receptor and they do not alter the activity of the soluble isoenzyme form of guanylate cyclase. Thus, the effects of atriopeptins are analogous in some respects to the effects of ST except that they alter cyclic GMP synthesis in numerous tissues due to the ubiquitous presence of the receptor.

Binding studies with radiolabeled atriopeptin analogues have given linear Scatchard plots with many tissue preparations, which suggests that a single class of receptors is present in most tissues. However, the comparison of peptide binding with cyclic GMP accumulation in our structure-activity studies clearly demonstrates that many tissues possess at least two distinct populations of binding sites (51-53). While the apparent affinities of these receptors to most atriopeptin analogues are similar (dissociation constant of 0.1 to $1 \mathrm{nM}$ ), the relative abundance of each receptor varies in different preparations. Cloned endothelial and smooth muscle cells also possess two classes of binding sites, indicating that the data are not due to cellular heterogeneity of preparations $(51,52)$. Interestingly, cross-linking studies with radiolabeled atriopeptins also reveal two binding sites that are $\sim 60,000$ and $120,000 \mathrm{D}$ in size $(54,55)$. Our current data indicate that only one of the receptors, designated ANF-1, is coupled to guanylate cyclase activation and cyclic GMP synthesis $(52,53,55)$. While the mechanism of atriopeptin receptor coupling to particulate guanylate cyclase activation is not known, we have found that the $120,000-\mathrm{D}$ binding site copurifies with particulate guanylate cyclase through numerous procedures (53). At present we suspect that ANF binding and cyclic GMP synthesis reside in the same membrane glycoprotein, and presumably this macromolecule spans the membrane (53).

Although atriopeptins bind to receptors on both endothelial cells and smooth muscle cells and increase cyclic GMP accu- 
mulation, their effects on cyclic GMP synthesis and relaxation of vascular smooth muscle are endothelium-independent (13, 45). Analogous to the nitrovasodilators, their effects are also independent of extracellular $\mathrm{Ca}^{++}$(13). The increase in cyclic GMP levels in vascular smooth muscle with atriopeptins is associated with increased activity of cyclic GMP-dependent protein kinase (56). While the vasorelaxant effects of the atriopeptins correlate with cyclic GMP synthesis, other effects of the atriopeptins can not be excluded. Furthermore, the role of the receptor that is not coupled to cyclic GMP synthesis designated ANF-2 is not presently known. It is of interest that atriopeptins have been found to inhibit adenylate cyclase activity in some preparations (57). The physiological relevance of this effect is unknown; nor is it known to which receptor this effect is coupled. It is unlikely that inhibition of cyclic AMP synthesis in vascular preparations with atriopeptins is associated with vasodilation since cyclic AMP synthesis, like cyclic GMP accumulation, correlates with vascular relaxation. While atriopeptins activate particulate guanylate cyclase in preparations of kidney, adrenal, and other tissues, additional studies are required to establish the roles of cyclic GMP, cyclic AMP, or perhaps other second messengers in the physiological effects of atriopeptins in these tissues. Although the marked effects of atriopeptins on cyclic GMP synthesis in endothelial cells suggest that other vascular effects of atriopeptins should be expected, the nature of these effects is also unknown. At present, it is almost certain that one class of atriopeptin receptors on vascular endothelial and smooth muscle cells is associated with the activation of particulate guanylate cyclase and the accumulation of cyclic $\operatorname{GMP}(52,55)$. Also, the accumulation of cyclic GMP with atriopeptins as with the effects of nitrovasodilators and endothelium-dependent vasodilators is associated with vascular relaxation. Since these three classes of vasodilators result in the activation of either the soluble or membrane-associated isoenzyme forms of guanylate cyclase and presumably increase different intracellular pools of cyclic GMP, some differences on vascular metabolism and function might be expected with additional studies. Although nitroglycerin-tolerant vessels have decreased cyclic GMP accumulation and relaxation when challenged with nitrovasodilators or endotheliumdependent vasodilators, the effects of atriopeptins are not altered (43). Interestingly, nitroglycerin-tolerant vessels have a relatively stable modification in the properties of soluble guanylate cyclase, while the particulate isoenzyme appears unaltered (58).

While cyclic GMP accumulation in vascular smooth muscle mediates the effects of these three classes of vasodilators, other effects of these agents should not be overlooked. Furthermore, other second messengers are known to participate in vasodilation induced with other vasorelaxants. Although the studies summarized here permit us to develop a framework for understanding the mechanism of action of these vasodilators (see Fig. 1), additional experiments are required to develop some innovative therapeutic approaches to cardiovascular disorders.

\section{Acknowledgments}

The author thanks Sherry Oppenheim and Beverly Lyons for typing the manuscript.

Some of the studies summarized in this review were supported with grants from the National Institutes of Health (AM 30787 and HL 28474), the Veterans Administration, and the Council for Tobacco ResearchUSA, Inc.

\section{References}

1. Murad, F., W. P. Arnold, C. K. Mittal, and J. M. Braughler. 1979. Adv. Cyclic Nucleotide Res. 11:175-204.

2. Mittal, C. K., and F. Murad. 1982. In Handbook of Experimental Pharmacology. Vol. 58-1. J. A. Nathanson and J. W. Kebabian, editors. Springer-Verlag, Berlin. 225-260.

3. Rapoport, R. M., and F. Murad. 1983. J. Cyclic Nucleotide Protein Phosphorylation Res. 9:281-296.

4. Murad, F., and G. D. Aurbach. 1978. In The Year in Metabolism. N. Freinkel, editor. Plenum Publishing Corp., New York. 1-32.

5. Arnold, W. P., C. K. Mittal, S. Katsuki, and F. Murad. 1977. Proc. Natl. Acad. Sci. USA. 74:3203-3207.

6. Mittal, C. K., and F. Murad. 1977. Proc. Natl. Acad. Sci. USA. 74:4360-4364.

7. Craven, P. A., F. R. DeRubertis, and D. W. Pratt. 1979. J. Biol. Chem. 254:8213-8222.

8. Ignarro, L. J., B. Ballot, and K. S. Wood. 1984. J. Biol. Chem. 259:6201-6207.

9. Hughes, J., F. Murad, B. Chang, and R. Guerrant. 1978. Nature (Lond.). 271:755-756.

10. Field, M., L. H. Graf, W. J. Laird, and P. L. Smith. 1978. Proc. Natl. Acad. Sci. USA. 75:2800-2804.

11. Guerrant, R. L., J. M. Hughes, B. Chang, D. C. Robertson, and F. Murad. 1980. J. Infect. Dis. 142:220-228.

12. Waldman, S. A., R. M. Rapoport, and F. Murad. 1984. J. Biol. Chem. 259:14332-14334.

13. Winquist, R. J., E. P. Faison, S. A. Waldman, K. Schwartz, F. Murad, and R. M. Rapoport. 1984. Proc. Natl. Acad. Sci. USA. 81: 7661-7664.

14. Waldman, S. A., M. S. Sinacore, J. A. Lewicki, L. Y. Chang, and F. Murad. 1984. J. Biol. Chem. 259:4038-4042.

15. Kimura, H., C. K. Mittal, and F. Murad. 1975. J. Biol. Chem. 250:8016-8022.

16. Mittal, C. K., H. Kimura, and F. Murad. 1977. J. Biol. Chem. 252:4348-4390.

17. Katsuki, S., W. Arnold, C. K. Mittal, and F. Murad. 1977. J. Cyclic Nucleotide Res. 3:23-25.

18. DeRubertis, F. R., and P. A. Craven. 1976. Science (Wash. DC). 193:897-899.

19. Kimura, H., C. K. Mittal, and F. Murad. 1975. Nature (Lond.). 275:700-702.

20. Katsuki, S., and F. Murad. 1977. Mol. Pharmacology. 13:330 341.

21. Schultz, K. D., K. Schultz, and G. Schultz. 1977. Nature (Lond.). 265:750-751.

22. Katsuki, S., W. P. Arnold, and F. Murad. 1977. J. Cyclic Nucleotide. Res. 3:239-247.

23. Diamond, J., and K. S. Blisard. 1976. Mol. Pharmacol. 12:688692.

24. Stryer, L. 1986. Annual Review of Neuroscience 9: In press.

25. Braughler, J. M., C. K. Mittal, and F. Murad. 1979. J. Biol. Chem. 254:12450-12454.

26. Brandwein, H. J., J. A. Lewicki, and F. Murad. 1981. J. Biol. Chem. 256:2958-2962.

27. Mittal, C. K., W. P. Arnold, and F. Murad. 1978. J. Biol. Chem. 253:1266-1271.

28. Gerzer, R., E. Bohme, F. Hoffman, and G. Schultz. 1981. FEBS (Fed. Eur. Biochem. Soc.) Lett. 123:71-74.

29. Kukovetz, W. R., S. Holzmann, A. Wurm, and G. Poch. 1979. Naunyn-Schmeideberg's Arch. Pharmacol. 310:129-138.

30. Pearl, R. G., M. H. Rosenthal, F. Murad, and J. A. Ashton. 1984. Anesthesiology. 61:712-715.

31. Rapoport, R. M., K. Schwartz, and F. Murad. 1985. Circulation Res. 57:164-170.

32. Fiscus, R. R., R. M. Rapoport, and F. Murad. 1983. J. Cyclic Nucleotide Protein Phosphorylation Res. 9:415-425. 
33. Fiscus, R. R., T. J. Torphy, and S. E. Mayer. 1984. Biochim. Biophys Acta. 805:382-392.

34. Rapoport, R. M., M. Draznin, and F. Murad. 1982. Proc. Natl. Acad. Sci. USA. 79:6470-6474.

35. Rapoport, R. M., M. Draznin, and F. Murad. 1983. Nature (Lond.). 306:174-176.

36. Rapoport, R. M., M. B. Draznin, and F. Murad. 1983. Trans. Assoc. Am. Physicians. 96:19-30.

37. Kobayashi, S., H. Kanaide, and M. Nakamura. 1985. Science (Wash. DC). 229:553-556.

38. Furchgott, R. F. 1984. Annu. Rev. Pharmacol. Toxicol. 24:175197.

39. Cocks, T. M., J. A. Angus, J. H. Campbell, and G. R. Campbell. 1985. J. Cell. Physiol. 123:310-320.

40. Rapoport, R. M., and F. Murad. 1983. Circ. Res. 52:352-357.

41. Rapoport, R. M., M. B. Draznin, and F. Murad. 1984. Circ. Res. 55:468-479.

42. Holzmann, S. 1982. J. Cyclic Nucleotide Res. 8:409-419.

43. Rapoport, R. M., S. A. Waldman, R. Ginsburg, C. Molina, and F. Murad. 1986. Eur. J. Pharmacol. In press.

44. Griffith, T. M., D. H. Edwards, M. J. Lewis, A. C. Newly, and A. H. Henderson. 1984. Nature (Lond.). 308:645-647.

45. Rapoport, R. M., S. A. Waldman, K. Schwartz, R. J. Winquist, and F. Murad. 1985. Eur. J. Pharmacol. 115:219-229.

46. DeBold, A. 1982. Proc. Soc. Exp. Biol. Med. 170:133-138.

47. Currie, M. G., D. M. Geller, B. R. Cole, J. G. Baylan, W. YuSheng,
S. W. Holmberg, and P. Needlemam. 1983. Science (Wash. DC). 221: 71-73.

48. DeLean, A., K. Racz, J. Gutkowska, T. T. Nguyen, M. Cantin, and J. Genest. 1984. Endocrinology. 115:1636-1638.

49. Nakayama, K., H. Ohkubo, T. Hirose, S. Inayama, and S. Nakanishi. 1984. Nature (Lond.). 310:699-701.

50. Yamanaka, M., B. Greenberg, L. Johnson, J. Seilhomer, M. Brewer, T. Friedmann, J. Miller, S. Atlas, J. Laragh, J. Lewicki, and F. Fiddes. 1984. Nature (Lond.). 309:719-722.

51. Leitman, D. C., S. A. Waldman, R. M. Rapoport, and F. Murad. 1985. Trans. Assoc. Am. Physicians. In press.

52. Leitman, D. C., and F. Murad. 1986. Biochim. Biophys. Acta. 885:74-79.

53. Kuno, T., J. W. Andresen, Y. Kamisaki, S. A. Waldman, L. Y. Chang, S. Saheki, D. C. Leitman, M. Nakane, and F. Murad. 1986. J. Biol. Chem. In press.

54. Vandlen, R. L., K. E. Arcuri., and M. A. Napier. 1985. J. Biol. Chem. 260:10889-10892.

55. Leitman, D. C., J. W. Andresen, T. Kuno, Y. Kamisaki, J. W. Chang, and F. Murad. J. Biol. Chem. In press.

56. Fiscus, R. R., R. M. Rapoport, S. A. Waldman, and F. Murad. 1985. Biochim. Biophys. Acta. 846:179-184.

57. Anand-Srivastava, M. B., D. J. Franks, M. Cantin, and J. Genest. 1984. Biochem. Biophys. Res. Commun. 121:855-862.

58. Waldman, S. A., R. M. Rapoport, R. Ginsburg, and F. Murad. 1986. Biochem. Pharmacol. In press. 